\title{
Analytical Solutions for Characterizing Fluid Flow through Sand-Pack in Pipes
}

\author{
Shichen Gao, ${ }^{1}$ Zhixin Tian, ${ }^{1}$ and Bailu Teng $\mathbb{D D}^{2}$ \\ ${ }^{1}$ School of Science, China University of Geosciences (Beijing), Beijing 100083, China \\ ${ }^{2}$ School of Energy Resources, China University of Geosciences (Beijing), Beijing 100083, China \\ Correspondence should be addressed to Bailu Teng; bailu@cugb.edu.cn
}

Received 28 March 2020; Revised 8 June 2020; Accepted 20 July 2020; Published 3 August 2020

Academic Editor: Jian G. Zhou

Copyright $(0) 2020$ Shichen Gao et al. This is an open access article distributed under the Creative Commons Attribution License, which permits unrestricted use, distribution, and reproduction in any medium, provided the original work is properly cited.

When fluid flows through a pipe that is packed with sand particles, the fluid will bear the resistance from the sand-pack, as well as the viscous shear from the pipe wall. If the viscous shear from the pipe wall can be neglected, the fluid flow will obey Darcy's law, and one can think the equivalent permeability of the packed-pipe equals the permeability of the sand-pack. However, if the viscous shear from the pipe wall cannot be neglected, the fluid flow will obey the Brinkman equation, and the permeability of the packedpipe will be less than that of the sand-pack due to the additional viscous drag. In this work, on the basis of the Brinkman equation, we derived a series of analytical solutions for characterizing the fluid flow in packed-pipes. These solutions can be used to depict the velocity profiles, estimate the flux rate, and calculate the equivalent permeability of a packed-pipe. On the basis of these analytical solutions, we found that Poiseuille's law is a special form of the derived equivalent permeability solution. We further divided the fluid flow in a packed-pipe into three regimes, including N-S flow, Brinkman flow, and Darcy flow. During the regime of Brinkman flow, the dimensionless flow velocity at the pipe center is 1 , and the dimensionless flow velocity is gradually decreased to 0 at the pipe wall. We also investigated the effects of sorting, sand particle size, and sand-pack porosity on the packed-pipe permeability. The calculated results show that a more uniform size of the sand particles or a smaller mean particle diameter can lead to lower packed-pipe permeability. Compared to the sorting and mean particle diameter, the sand-pack porosity exerts a more significant effect on the packed-pipe permeability.

\section{Introduction}

The Navier-Stokes (N-S) equation describes fluid flow on the microlevel and it accounts for the effect of viscous shear on the fluid flow $[1,2]$. For an incompressible fluid, neglecting the inertial terms, the N-S can be reduced to

$$
\nabla p=\frac{\mu}{\beta_{1} \beta_{2} \phi} \nabla^{2} v
$$

where $p$ is the pressure ( $\mathrm{MPa}), \mu$ is the viscosity (mPa.s), $\beta_{1}$ and $\beta_{2}$ are the unit conversion factors, $\nu$ is the velocity $(\mathrm{m} / \mathrm{d})$, and $\phi$ is the porosity. On the basis of the reduced N-S equation, Hagenbach [3] derived an analytical solution for characterizing the flux rate in empty pipes (in this work, an empty pipe indicates a pipe that is filled only with fluid), which can be expressed as

$$
q=\frac{\beta_{1} \beta_{2} \pi R^{4}}{8 \mu} \frac{\Delta p}{L},
$$

where $q$ is the flux rate $\left(\mathrm{m}^{3} / \mathrm{d}\right), R$ is the inner radius of the pipe (m), and $L$ is the length of the pipe (m). Equation (2) is also widely known as the Poiseuille-Hagenbach equation. Figure 1(a) shows the velocity profile of fluid flow in an empty pipe. From Figure 1(a), one can find that the fluid exhibits small flow velocity at the pipe wall due to the effect of viscous shear. In addition, if we use equivalent permeability to characterize the ability of the empty pipes for transmitting the fluid, on the basis of Darcy's law [4] we can have

$$
q=\frac{\beta_{1} \pi R^{2} k_{e}}{\mu} \frac{\Delta p}{L}
$$




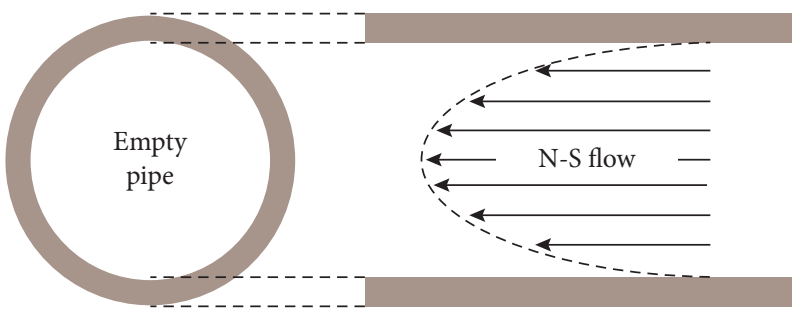

(a)

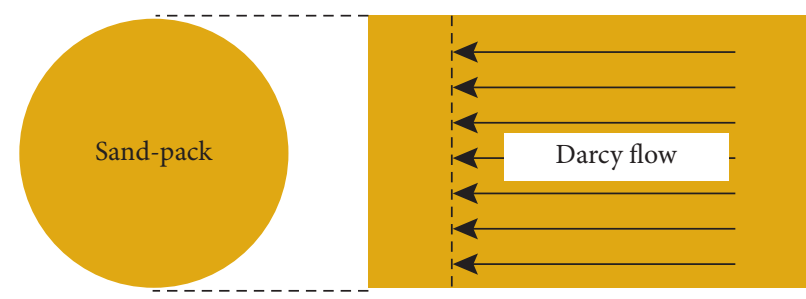

(b)

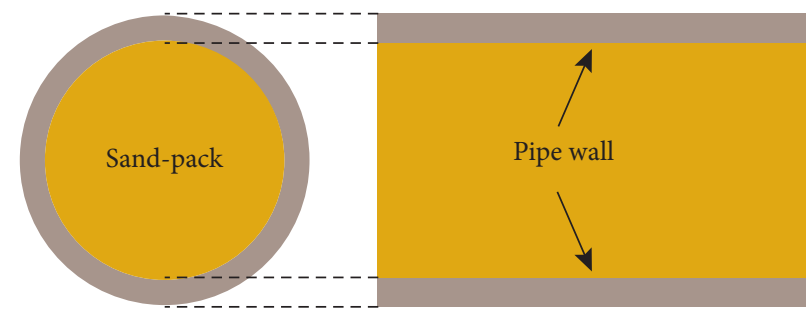

(c)

Figure 1: Fluid flow in pipes, sand-packs, and packed-pipes: (a) velocity profile of fluid flow in an empty pipe; (b) velocity profile of fluid flow in a cylinder sand-pack; (c) a pipe that is packed with sand.

where $k_{e}$ is the equivalent permeability $(\mathrm{mD})$. Substituting equation (2) into equation (3) gives

$$
k_{e}=\frac{\beta_{2} R^{2}}{8} \text {. }
$$

Equation (4) describes the relationship between the equivalent permeability of the empty pipe and the inner radius of pipes. This equation is commonly called Poiseuille's law.

Darcy's law characterizes the fluid flow on the macrolevel, and it considers the resistance from porous media on the fluid flow. If fluid flows through a cylinder sand-pack whose radius is $R$ and whose length is $L$, the flux rate can also be calculated with Darcy's law (i.e., Equation (3)). The only difference is that the equivalent permeability in Equation (3) should equal the sand-pack permeability:

$$
k_{e}=k_{s},
$$

where $k_{s}$ is the permeability of sand-pack $(\mathrm{mD})$. Figure $1(\mathrm{~b})$ illustrates the velocity profile of Darcy flow in the cylinder sand-pack. On the macrolevel, one can think that the velocity of Darcy flow is uniform along a given cross-section.

According to the aforementioned arguments, one can find that we can use analytical methods to describe the fluid flow in empty pipes or sand-packs. However, how to calculate the flux rate through a pipe that is packed with sand (see Figure 1(c)) is investigated. It should be noted that, for the scenario of Figure 1(a), the fluid flow is only affected by the viscous shear from the pipe wall, whereas, for the scenarios of Figure 1(b), the fluid flow is only affected by the effect of the sand-pack. Therefore, if fluid flows through a packed-pipe, both the viscous shear and the sand-pack will exert their effects on the fluid flow.
In recent years, various studies have been conducted to investigate the fluid flow in packed-pipes. Chen et al. [5] developed a momentum equation for characterizing the fluid flow in a packed tube by combining the N-S equation, Darcy's law, and a superficial dispersion term due to phase discontinuity. Yuki et al. [6] utilized a matched refractiveindex method to perform a visualization investigation on the flow structure in a sphere-packed-pipe. Three flows are observed in their experiment, including bypass flow, secondary flow, and spouting flow. Siddiqui et al. [7] developed an analytical solution to characterize the fluid flow through the porous media between two coaxial cylinders. In their study, the inner cylinder is stationary, while the outer cylinder travels parallel to itself. Hashim and Kamel [8] proposed a new correlation method for calculating the pressure drop through a packed-pipe. Their method is validated by comparing the results of their work to experimental data that are measured in a horizontal packed-pipe with homogeneous spherical pellets. Wu et al. [9] studied the Taylor dispersion in a packed-pipe by accounting for the effect of wall reaction. Their study is based on the method of Gill's series solution. More studies about fluid flow in packedpipes can be found in Nazififard and Suh [10], Wu et al. [9], and Yang et al. [11]. Although these studies provided us with a comprehensive insight into the fluid flow in packed-pipes, these works are mainly conducted with numerical methods, which are highly computationally demanding. Therefore, it is imperative to derive analytical solutions for characterizing such kind of flow.

In practice, one kind of utilizations of the packed-pipes is to conduct sand-pack permeability tests. In these tests, it is commonly assumed that the viscous shear from the pipe wall can be neglected. As such one can think that the sand-pack 
permeability equals the measured permeability. However, if the permeability of the sand-pack is sufficiently large or the diameter of the pipe is sufficiently small, the viscous shear from the pipe cannot be neglected. In such cases, it is not reasonable to approximate the sand-pack permeability with the packed-pipe permeability.

To account for the effect of viscous shear on the fluid flow in porous media, Brinkman [12] proposed a new equation by combining the reduced N-S equation with Darcy's law. This equation is subsequently named as Brinkman equation, which is given as

$$
\nabla p=\frac{\mu}{\beta_{1} \beta_{2} \phi} \nabla^{2} \mathbf{v}-\frac{\mu}{\beta_{1} k_{s}} \mathbf{v}
$$

In equation (6), the fluid is incompressible, the flow is assumed to be laminar, and the inertial force is neglected. It is worth noting that equation (6) is different from the original Brinkman equation by considering the effect of volume fraction of solid in porous media. The original Brinkman equation ignored the effect of the volume fraction of solid; hence, it will induce significant error if the solid volume fraction is sufficiently high or $\phi$ is sufficiently small [13-16]; and [17]. For the sake of convenience, although equation (6) is a modified form of the original Brinkman Equation, in this work, we still call it Brinkman equation. With the aid of equation (6), we derived analytical solutions for characterizing the fluid flow in packed-pipes. On the basis of the analytical solutions, we depicted the velocity profiles of the fluid flow in packed-pipes. In addition, we conducted a comprehensive comparison between the equivalent permeability of the packed-pipes and that of the sand-pack.

\section{Methodology}

In order to obtain analytical solutions for characterizing the fluid flow through packed-pipes, we made the following assumptions:

(1) The fluid flow is laminar and is in steady-state

(2) The properties of the fluid and sand-pack are constant

(3) The sand-pack is homogeneous and the pipe has a uniform diameter

(4) There is no slippage at the pipe wall

(5) The effect of gravity is neglected

According to the above assumptions, the parameters of $\mu, \phi$, and $k_{s}$ in equation (6) are constant. In addition, in a cylindrical coordinate system, equation (6) can be rewritten as

$$
\nabla p=\frac{\mu}{\beta_{1} \beta_{2} \phi} \frac{1}{r} \frac{\mathrm{d}}{\mathrm{d} r}\left(r \frac{\mathrm{d} v}{\mathrm{~d} r}\right)-\frac{\mu}{\beta_{1} k_{s}} v,
$$

where $r$ is along the direction from the pipe center to the pipe wall (m). Rearranging equation (7) gives

$$
\frac{\mathrm{d}^{2} v}{\mathrm{~d} r^{2}}+\frac{1}{r} \frac{\mathrm{d} v}{\mathrm{~d} r}-\frac{\beta_{2} \phi}{k_{s}} v=\frac{\beta_{1} \beta_{2} \phi}{\mu} \nabla p .
$$

Equation (8) is a generalized Bessel equation whose general solution is

$$
v(r)=A I_{0}\left(\sqrt{\frac{\beta_{2} \phi}{k_{s}}} r\right)+B K_{0}\left(\sqrt{\frac{\beta_{2} \phi}{k_{s}}} r\right)-\frac{\beta_{1} k_{s}}{\mu} \nabla p,
$$

where $I_{n}$ indicates the $n^{\text {th }}$ order modified Bessel function of the first kind, $K_{n}$ indicates the $n^{\text {th }}$ order modified Bessel function of the second kind, and $A$ and $B$ are constants that require to be determined. At the pipe center, we have $r=0$ and

$$
B K_{0}\left(\sqrt{\frac{\beta_{2} \phi}{k_{s}}} r\right)=B K_{0}(0)=\infty .
$$

Since the flow velocity should not have an infinite value at $r=0$, the constant $B$ should equal 0 , and equation (9) can be reduced to

$$
v(r)=A I_{0}\left(\sqrt{\frac{\beta_{2} \phi}{k_{s}}} r\right)-\frac{\beta_{1} k_{s}}{\mu} \nabla p .
$$

As we have assumed that there is no slippage at the pipe wall, we can have

$$
v(r=R)=0 .
$$

Inserting equation (11) into equation (12) yields

$$
A=\frac{1}{I_{0}\left(\sqrt{\left(\beta_{2} \phi / k_{s}\right) R}\right)} \frac{\beta_{1} k_{s}}{\mu} \nabla p
$$

Inserting equation (13) into equation (11) gives the equation characterizing the velocity profile:

$$
v(r)=\left[\frac{I_{0}\left(\sqrt{\left(\beta_{2} \phi / k_{s}\right) r}\right)}{I_{0}\left(\sqrt{\left(\beta_{2} \phi / k_{s}\right) R}\right)}-1\right] \frac{\beta_{1} k_{s}}{\mu} \nabla p .
$$

Equation (14) is in the format of a vector. Rewriting equation (14) in the format of scalar, we can have

$$
v(r)=\left[1-\frac{I_{0}\left(\sqrt{\left(\beta_{2} \phi / k_{s}\right) r}\right)}{I_{0}\left(\sqrt{\left(\beta_{2} \phi / k_{s}\right) R}\right)}\right] \frac{\beta_{1} k_{s}}{\mu} \frac{\Delta p}{L} .
$$

The flux rate $q$ can be calculated as follows:

$$
\begin{aligned}
q & =\int_{r=0}^{r=R} 2 \pi r v(r) d r=\int_{r=0}^{r=R} 2 \pi r\left[1-\frac{I_{0}\left(\sqrt{\left(\beta_{2} \phi / k_{s}\right) r}\right)}{I_{0}\left(\sqrt{\left(\beta_{2} \phi / k_{s}\right) R}\right)}\right] \frac{\beta_{1} k_{s}}{\mu} \frac{\Delta p}{L} \mathrm{~d} r \\
& =\frac{\pi R^{2} \beta_{1} k_{s}}{\mu} \frac{\Delta p}{L}\left[1-\frac{2}{R} \sqrt{\frac{k_{s}}{\beta_{2} \phi_{p}}} \frac{I_{1}\left(\sqrt{\left(\beta_{2} \phi / k_{s}\right) R}\right)}{I_{0}\left(\sqrt{\left(\beta_{2} \phi / k_{s}\right) R}\right)}\right] .
\end{aligned}
$$


Comparing equation (16) to Darcy's law (i.e., Equation (3)), we can obtain the relationship between the equivalent permeability of packed-pipe and the permeability of sandpack:

$$
k_{e}=k_{s}\left[1-\frac{2}{R} \sqrt{\frac{k_{s}}{\beta_{2} \phi}} \frac{I_{1}\left(\sqrt{\left(\beta_{2} \phi / k_{s}\right) R}\right)}{I_{0}\left(\sqrt{\left(\beta_{2} \phi / k_{s}\right) R}\right)}\right] .
$$

Equations (15) through (17) are the equations that characterize the fluid flow in packed-pipes.

Particularly, for an empty pipe, we can think it is packed with a sand-pack whose permeability is infinite and whose porosity is 1 . Thus, the term $\sqrt{\left(\beta_{2} \phi / k_{s}\right)}$ in equation (17) approaches 0 . On the basis of Taylor series, expanding $I_{1}$ and $I_{0}$ at 0 and using the first two terms of the series to approximate the results, we can have

$$
\begin{aligned}
k_{e} & =k_{s}\left[1-\frac{2}{R} \sqrt{\frac{k_{s}}{\beta_{2} \phi}} \frac{I_{1}\left(\sqrt{\left(\beta_{2} \phi / k_{s}\right) R}\right)}{I_{0}\left(\sqrt{\left(\beta_{2} \phi / k_{s}\right) R}\right)}\right] \\
& \approx k_{s}\left\{1-\frac{2}{R} \sqrt{\frac{k_{s}}{\beta_{2} \phi}}\left[\frac{\left.(1 / 2)\left(\sqrt{\left(\beta_{2} \phi / k_{s}\right) R}\right)+(1 / 16)\left(\sqrt{\left(\beta_{2} \phi / k_{s}\right) R}\right)^{3}\right]}{1+(1 / 4)\left(\sqrt{\left(\beta_{2} \phi / k_{s}\right) R}\right)^{2}}\right]\right\} \\
& =k_{s}\left\{1-\frac{2}{R} \sqrt{\frac{k_{s}}{\beta_{2} \phi}}\left[\frac{1}{2}\left(\sqrt{\frac{\beta_{2} \phi}{k_{s}}} R\right)+\frac{1}{16}\left(\sqrt{\frac{\beta_{2} \phi}{k_{s}}} R\right)^{3}\right]\right\} \\
& =k_{s}\left\{1-1+\frac{\beta_{2} \phi R^{2}}{8 k_{s}}\right\} \\
& =\frac{\beta_{2} \phi R^{2}}{8} .
\end{aligned}
$$

The truncation errors of the Taylor series of $I_{1}$ and $I_{0}$ are at the order of $\left(\sqrt{\left(\beta_{2} \phi / k_{s}\right) R}\right)^{5}$ and $\left(\sqrt{\left(\beta_{2} \phi / k_{s}\right) R}\right)^{4}$, respectively. For empty pipes, we have $\phi=1$. Inserting $\phi=1$ into equation (18), equation (18) will be reduced to equation (4). In addition, if the radius of the pipe is sufficiently large, we will have

$$
\begin{aligned}
k_{e} & =k_{s}\left[1-\frac{2}{R} \sqrt{\frac{k_{s}}{\beta_{2} \phi}} \frac{I_{1}\left(\sqrt{\left(\beta_{2} \phi / k_{s}\right) R}\right)}{I_{0}\left(\sqrt{\left(\beta_{2} \phi / k_{s}\right) R}\right)}\right] \\
& \approx k_{s}\{1-0\} \\
& =k_{s} .
\end{aligned}
$$

This implies that both equations (4) and (5) are special forms of equation (17).

\section{Validation}

In order to validate the derived analytical solutions, we compared the permeability that is calculated with equation (17) to the permeability that is calculated from the

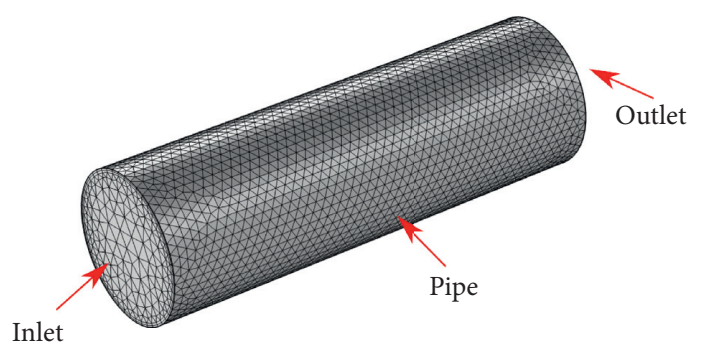

Figure 2: The model that is used for validation purposes.

commercial software [18]. The model that is used for validation is shown in Figure 2. Figure 2 indicates a pipe that is filled with porous media. The model built in COMSOL has the same assumptions to the model used in this work. These assumptions can be found in the section of methodology. The permeability of the porous media $\left(k_{s}\right)$ is $1 \times 10^{7} \mathrm{mD}$, the porosity of the porous media $(\phi)$ is 0.1 , the radius of the pipe $(R)$ is varied from $0.001 \mathrm{~m}$ to $0.1 \mathrm{~m}$, the length of the pipe $(L)$ is $0.1 \mathrm{~m}$, the flow velocity $(v)$ is set to be $8640 \mathrm{~m} / \mathrm{d}$, and the viscosity of the fluid $(\mu)$ is $1 \mathrm{mPa}$.s. The fluid flows into the pipe through the inlet surface and flows out of the pipe through the outlet surface. The equivalent permeability of the packed-pipe can be calculated based on Darcy's law if the pressure difference between the inlet surface and outlet surface is measured. With the aid of COMSOL, we measured the average pressure at the inlet surface and the outlet surface and subsequently calculated the equivalent permeability of the packed-pipe with the measured data. The results are summarized in Table 1.

Taking the case of $R=0.001 \mathrm{~m}$ as an example, the equivalent permeability can be calculated as follows:

$$
\begin{aligned}
k_{e} & =\frac{v \mu L}{\beta_{1}\left(p_{\text {in }}-p_{\text {out }}\right)}=\frac{8640 \times 1 \times 0.1}{0.0853 \times\left(2021.3 \times 10^{-6}-1.5582 \times 10^{-6}\right)} \\
& =5014975.985 \mathrm{~m} \mathrm{D.} .
\end{aligned}
$$

In addition, the equivalent permeability can be calculated with equation (17); thus, we can validate the proposed solution by comparing the results from this work to those from COMSOL. Figure 3 shows the comparison. In this figure, one can find that although the results from COMSOL are slightly larger than those from the proposed solution, these two plots show a similar trend. Actually, the larger values from COMSOL can be ascribed to numerical effects. In COMSOL, the modified Brinkman equation is solved with the finite element method, which will induce numerical errors during the computation. For example, for the case of $R=0.1 \mathrm{~m}$ in Table 1 , the calculated equivalent permeability $k_{e}$ is $10005636.16 \mathrm{mD}$, which is even larger than the permeability of the sand-pack $\left(k_{s}=1 \times 10^{7} \mathrm{mD}\right)$. However, in practice, the equivalent permeability of the packed-pipe should be less than the permeability of the sand-pack due to the additional viscous shear from the pipe wall. This indicates that the permeability of the packed-pipe has been 
TABLE 1: Pressures that are measured in COMSOL and the calculated permeability.

\begin{tabular}{lcrc}
\hline Inner radius of the pipe $(R, \mathrm{~m}))$ & $\begin{array}{c}\text { Average pressure at the inlet surface } \\
\left(p_{\text {in }}, \mathrm{MPa}\right)\end{array}$ & $\begin{array}{c}\text { Average pressure at the outlet surface } \\
\left(p_{\text {out }}, \mathrm{MPa}\right)\end{array}$ & $\begin{array}{c}\text { Equivalent permeability } \\
\left(k_{e}, \mathrm{mD}\right)\end{array}$ \\
\hline 0.0010 & $2021.3 \times 10^{-6}$ & $1.5582 \times 10^{-6}$ & 5014975.985 \\
0.0025 & $1287.7 \times 10^{-6}$ & $0.6038 \times 10^{-6}$ & 7869619.177 \\
0.0063 & $1103.2 \times 10^{-6}$ & $0.2497 \times 10^{-6}$ & 9183511.206 \\
0.0158 & $1041.4 \times 10^{-6}$ & $0.1566 \times 10^{-6}$ & 9727750.627 \\
0.0398 & $1015.4 \times 10^{-6}$ & $0.3740 \times 10^{-6}$ & 9979012.383 \\
0.1000 & $1012.9 \times 10^{-6}$ & $0.5749 \times 10^{-6}$ & 10005636.16 \\
\hline
\end{tabular}

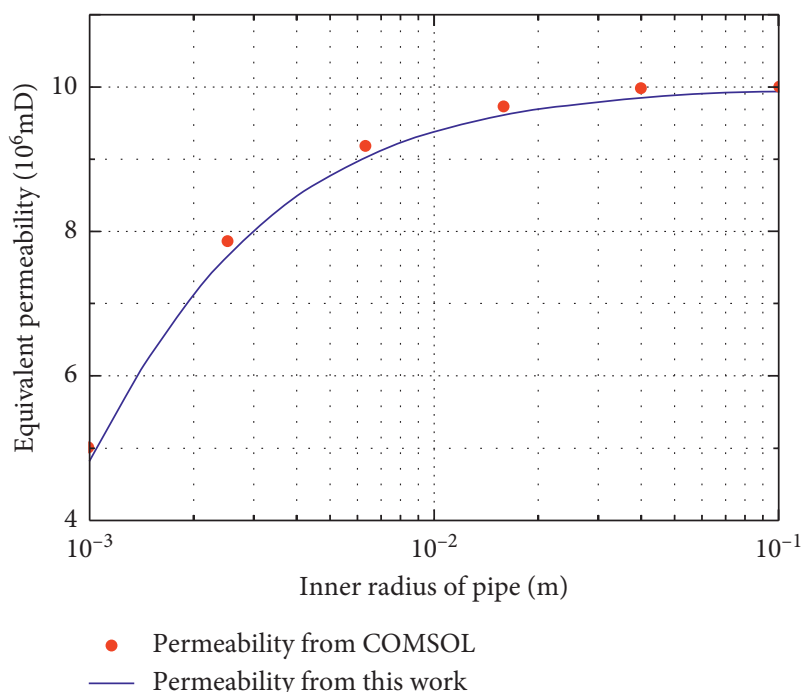

FIGURE 3: Comparison between the results from COMSOL and that from this work.

overestimated by COMSOL because of the effect of numerical errors.

\section{Results and Discussion}

In this section, we recognized the flow regimes in packedpipes and depicted the velocity profiles of these flow regimes. In addition, we conducted a comprehensive sensitivity analysis to study the effects of sand-pack properties on the packed-pipe permeability. For the sake of convenience, we define the following dimensionless parameters:

$$
\begin{gathered}
r_{D}=\frac{r}{R}, \\
v_{D}=\frac{v \mu L}{\beta_{1} k_{s} \Delta p}, \\
D_{a}=\sqrt{\frac{\beta_{2} \phi}{k_{s}}} R, \\
k_{r}=\frac{k_{e}}{k_{s}},
\end{gathered}
$$

where $r_{D}$ is the dimensionless radius, $v_{D}$ is the dimensionless velocity, $D_{a}$ is the Darcy parameter, and $k_{r}$ is the permeability ratio. Inserting equations (21) through (24) into equations (15) and (17) gives

$$
\begin{aligned}
v_{D}\left(r_{D}\right) & =1-\frac{I_{0}\left(D_{a} r_{D}\right)}{I_{0}\left(D_{a}\right)}, \\
k_{r} & =1-\frac{2}{D_{a}} \frac{I_{1}\left(D_{a}\right)}{I_{0}\left(D_{a}\right)} .
\end{aligned}
$$

4.1. Flow Regimes. Figure 4 shows the value of $k_{r}$ with different values of $D_{a}\left(D_{a}\right.$ is varied from 1 to 1000) in a loglog plot. From this figure, one can find that the permeability ratio is increased as the Darcy parameter $D_{a}$ is increased. At large value of $D_{a}$, the permeability ratio $k_{r}$ approaches 1 , which indicates Darcy flow. At small values of $D_{a}, k_{r}$ can be much smaller than 1 , which implies that the equivalent permeability of the packed-pipe is much smaller than the permeability of the sand-pack.

Figure 5 illustrates the equivalent permeability of the packed-pipe with a constant value of sand-pack permeability $\left(k_{s}=1 \times 10^{7} \mathrm{mD}\right)$, a constant value of sand-pack porosity $(\phi=0.1)$, and different values of pipe radius. In Figure 5, it can be observed that, at small value of pipe radius, the equivalent permeability approaches the results of

$$
k_{e}=\frac{\beta_{2} \phi R^{2}}{8},
$$

whereas at large value of pipe radius, the equivalent permeability approaches the results of equation (5) (i.e., $k_{e}=k_{s}$ ). According to Figure 5, one can divide the fluid flow into three regimes, including N-S flow, Brinkman flow, and Darcy flow.

N-S flow: at small pipe radius, the fluid flow in a packedpipe is mainly influenced by the effect of the viscous shear from pipe wall, and the sand-pack exerts its effect through its volume fraction. The equivalent permeability of pack-pipes approaches the results of equation (27) during this regime; Darcy flow: at large value of pipe radius, the viscous shear from pipe wall can be neglected, and the fluid flow is mainly influenced by the resistance from the sand-pack. During this regime, the equivalent permeability of the pack-pipe approaches the permeability of sand-pack; Brinkman flow: this flow regime is in a transition between N-S flow and Darcy flow. During this flow regime, both the viscous shear from pipe wall and the resistance from the sand-pack can significantly influence the fluid flow.

Figure 6 presents the equivalent permeability of packed pipe as a function of sand-pack permeability with a constant pipe radius of $5 \times 10^{-3} \mathrm{~m}$ and a constant sand-pack porosity of 


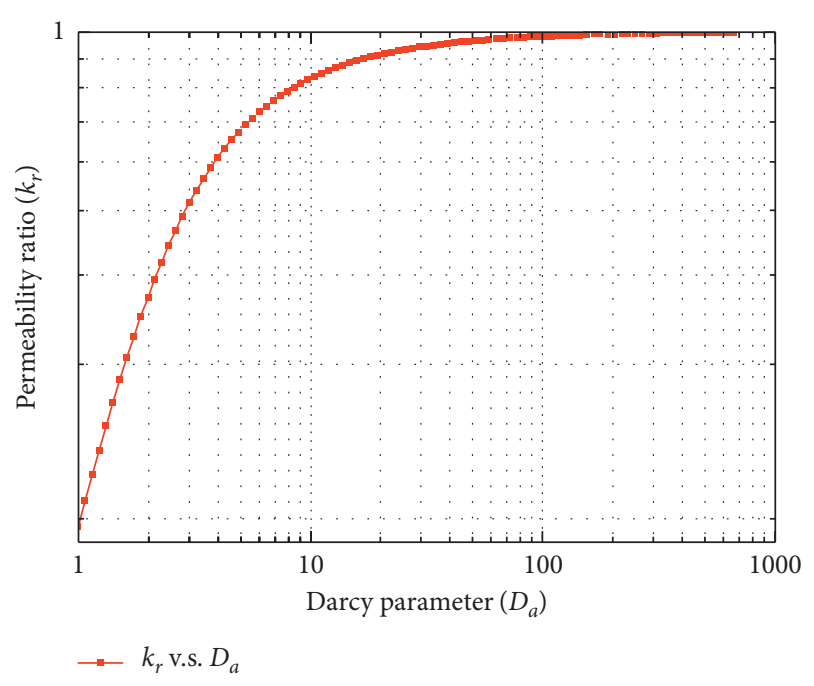

Figure 4: Permeability ratio with different values of $D_{a}$.

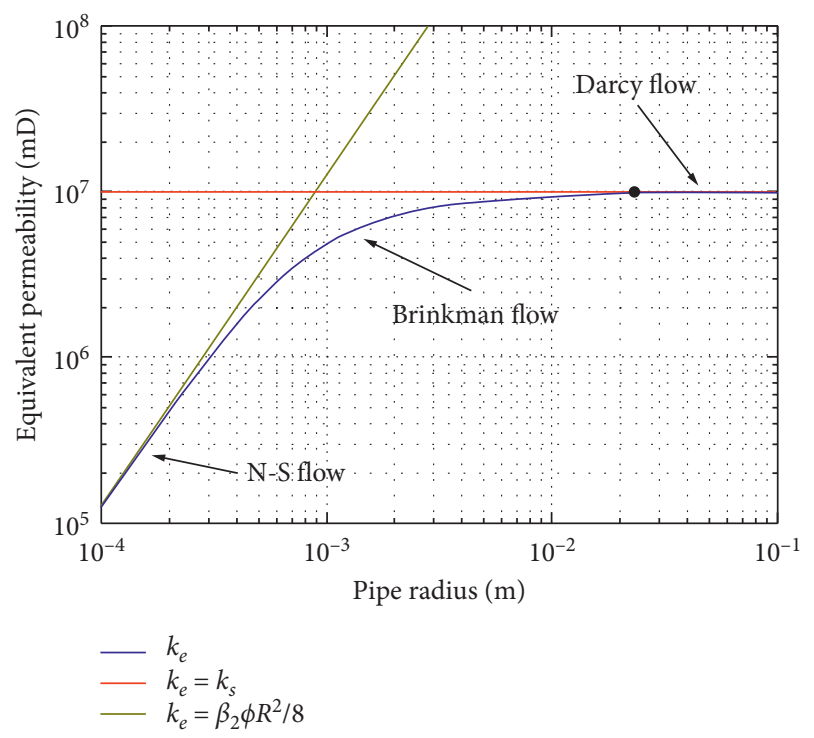

FIGURE 5: Equivalent permeability of packed-pipe as a function of pipe radius.

0.1. In Figure 6, one can find that the fluid flow can also be divided into three flow regimes. These flow regimes can be recognized as Darcy flow, Brinkman flow, and N-S flow as the sand-pack permeability is increased.

It should be noted that, due to the fact that the N-S flow and Darcy flow can be regarded as two extreme scenarios of Brinkman flow, the division of the flow regimes in Figures 5 and 6 is somehow qualitative rather than rigorously defined. The N-S flow is Brinkman flow neglecting the resistance from the sand-pack, whereas the Darcy flow is Brinkman flow neglecting the viscous shear from the pipe wall. In this work, to make a clear division between these three flow regimes, a threshold value $\varepsilon$ of permeability relative difference is assigned:

$$
\varepsilon=\left|\frac{K_{e}-k_{e}}{k_{e}}\right| \times 100 \%,
$$

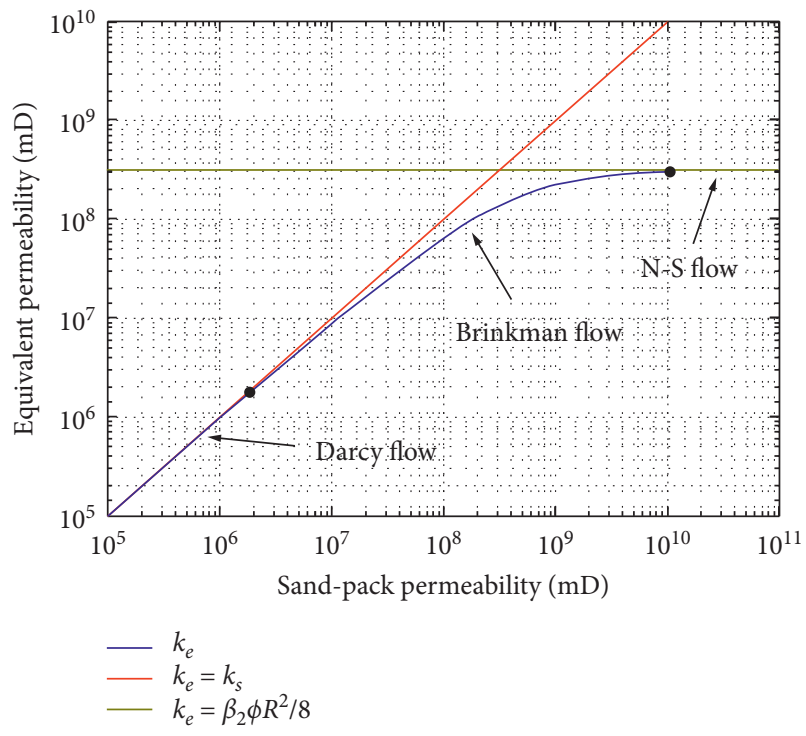

FIGURE 6: Equivalent permeability of packed-pipe as a function of sand-pack permeability.

where $K_{e}$ is the equivalent permeability from equation (27) or (5) and $k_{e}$ is the equivalent permeability of the packedpipe that is calculated with equation (17). Inserting equations (5), (17), (21), (22), (23), and (27) into equation (28), if $K_{e}$ is obtained with equation (27), we can have

$$
\varepsilon=\left|\frac{D_{a}^{2}}{8\left[1-\left(2 / D_{a}\right)\left(I_{1}\left(D_{a}\right) / I_{0}\left(D_{a}\right)\right)\right]}-1\right| \times 100 \%,
$$

and if $K_{e}$ is obtained with equation (5), we can have

$$
\varepsilon=\left|\frac{2 I_{1}\left(D_{a}\right)}{D_{a} I_{0}\left(D_{a}\right)-2 I_{1}\left(D_{a}\right)}\right| \times 100 \% .
$$

Taking 5\% as the threshold value, in this work, if $\varepsilon \leq 5 \%$, we can think the flow regime is N-S flow or Darcy flow. Inserting $\varepsilon \leq 5 \%$ into equation (29) and (30) yields

$$
D_{a} \leq 0.5481
$$

for equation (29) and

$$
D_{a} \geq 41.3899,
$$

for equation (30). This implies that the flow regime can be regarded as N-S flow if $D_{a} \leq 0.5481$, and as Darcy flow if $D_{a} \geq 41.3899$.

4.2. Velocity Profile. In this section, we studied the velocity profiles in a packed-pipe by varying the Darcy parameter $D_{a}$ from 0.1 to 100 . The velocity profiles of N-S flow regime, Brinkman flow regime, and Darcy flow regime are presented in Figures 7-9, respectively. Figure 7 shows the velocity profiles of N-S flow with $D_{a}=0.1,0.2,0.3,0.4$, and 0.5 . As defined in equation (25), $v_{D}=1$ indicates Darcy flow. In this figure, one can find that, for N-S flow regime, the dimensionless velocity has been significantly jeopardized due to the effect of viscous shear from pipe wall. The highest 


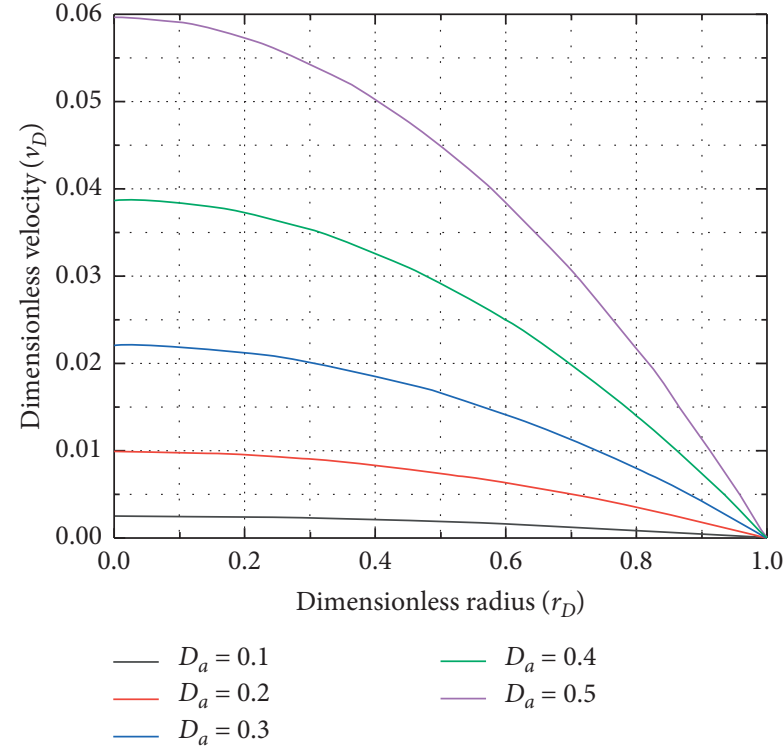

Figure 7: Dimensionless flow velocity of N-S flow regime with different values of $D_{a}$.

dimensionless velocity can be observed at the pipe center. As $D_{a}$ is increased, the dimensionless velocity is also increased.

Figure 8 illustrates the dimensionless velocity profiles during the Brinkman flow regime in a packed-pipe with $D_{a}=8,16,24,32$, and 40 . In this figure, it can be observed that the dimensionless velocity approaches the value of 1 at the pipe center, which indicates the fluid flow approaches Darcy flow. A smooth transition can be found between $r_{D}=0$ and $r_{D}=1$. During this transition, the dimensionless velocity is decreased from $v_{D}=1$ to $v_{D}=0$ because of the effect of viscous shear from pipe wall. In Figure 8, one can find that the Brinkman flow is a combination of Darcy flow near the pipe center and the N-S flow near the pipe wall. Figure 9 shows the dimensionless velocity profiles of Darcy flow regime with $D_{a}=60,70,80,90$, and 100 . In this figure, the transition is very short, and the dimensionless velocity is rapidly decreased to 0 at the pipe wall. In these plots, $v_{D}=1$ accounts for a large proportion, and the fluid flow can be regarded to be Darcy flow.

4.3. Sensitivity Analysis. In this section, we conducted a thorough sensitivity analysis on the packed-pipe permeability with different sand-pack properties. Berg [19] built the relationship between the sand-pack permeability and the sand-pack properties, which is given as

$$
k_{s}=80.8 \times 10^{12} e^{-1.385 \gamma} D^{2} \phi^{5.1},
$$

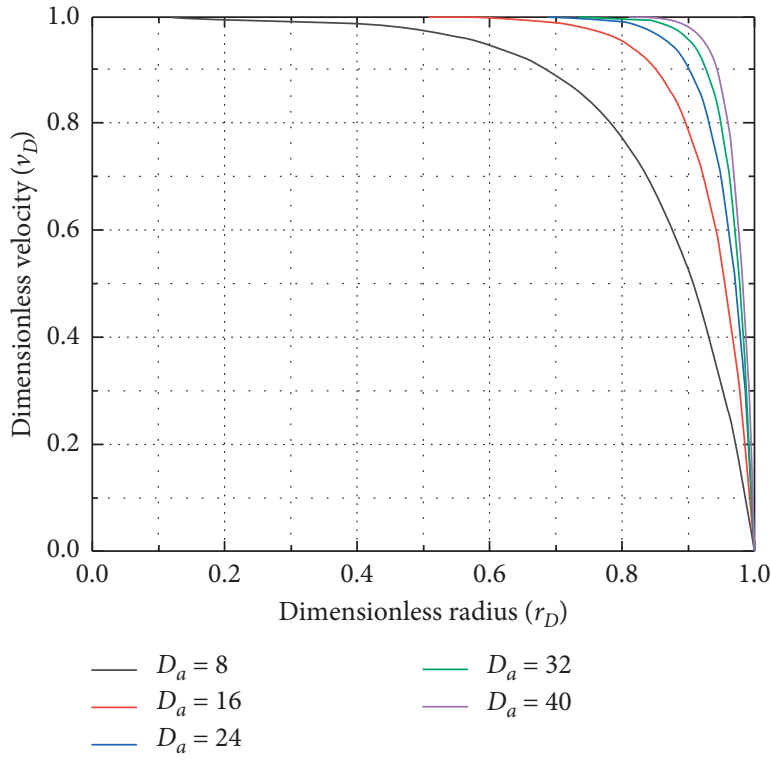

FIgURE 8: Dimensionless flow velocity of Brinkman flow regime with different values of $D_{a}$.

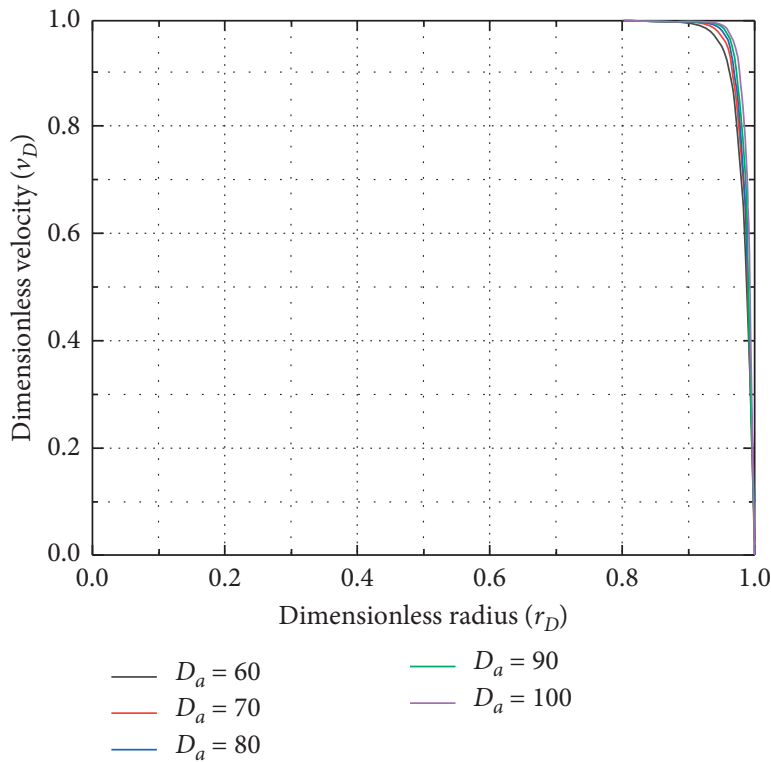

FIGURE 9: Dimensionless flow velocity of Darcy flow regime with different values of $D_{a}$.

where $\gamma$ is the sorting term in phi unit and $D$ is the geometric mean diameter of the sand particle. Inserting equation (33) into equation (17) yields

$$
k_{e}=80.8 \times 10^{12} e^{-1.385 \gamma} D^{2} \phi^{5.1} \times\left[1-\frac{2}{R} \sqrt{0.08 e^{-1.385 \gamma} D^{2} \phi^{4.1}} \frac{I_{1}\left(\sqrt{12.5 e^{1.385 \gamma} D^{-2} \phi^{-4.1}} R\right)}{I_{0}\left(\sqrt{12.5 e^{1.385 \gamma} D^{-2} \phi^{-4.1}} R\right)}\right] .
$$




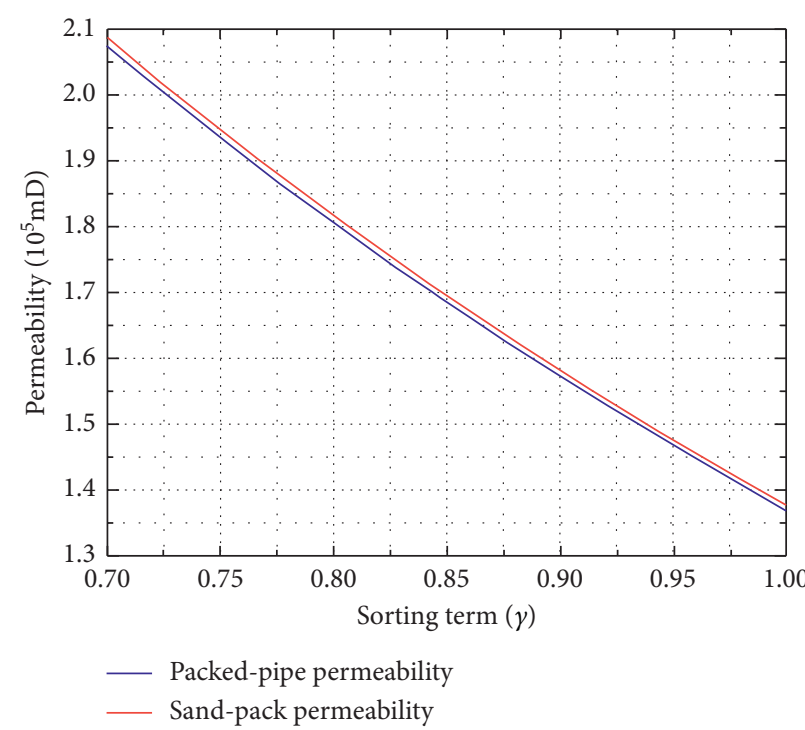

(a)

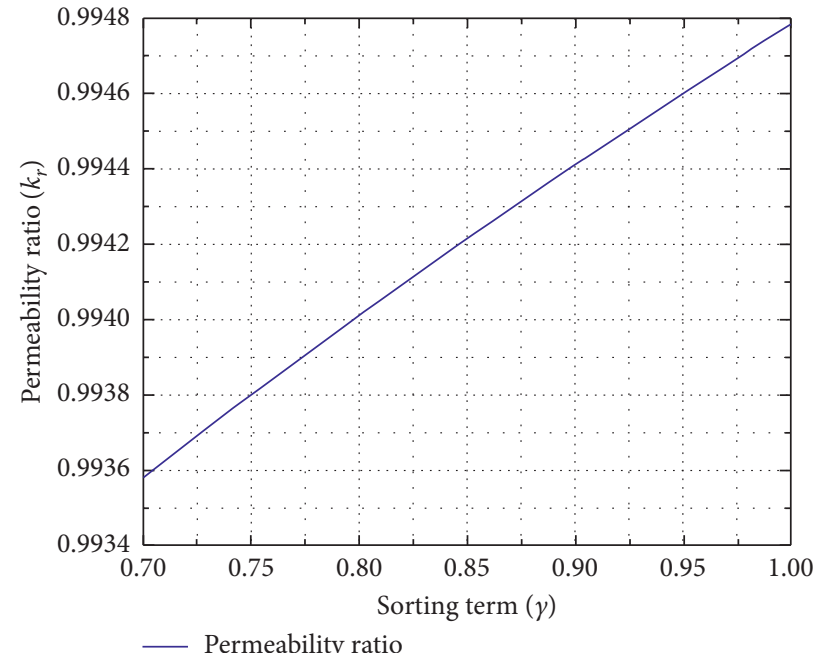

(b)

Figure 10: Packed-pipe permeability, sand-pack permeability, and permeability ratio as a function of sorting term $\gamma$ : (a) packed-pipe permeability and sand-pack permeability as a function of sorting term $\gamma$; (b) permeability ratio as a function of sorting term $\gamma$.

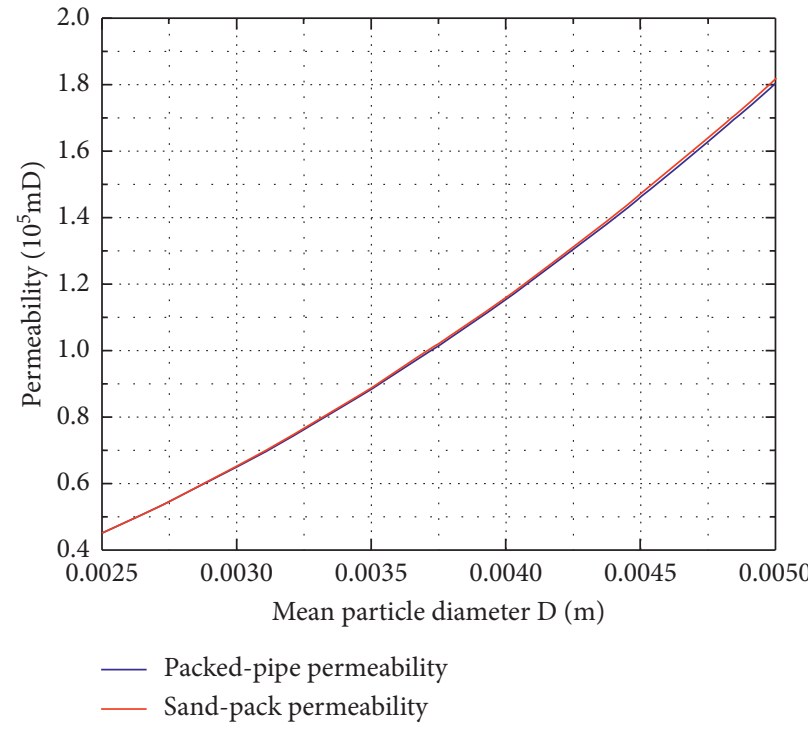

(a)

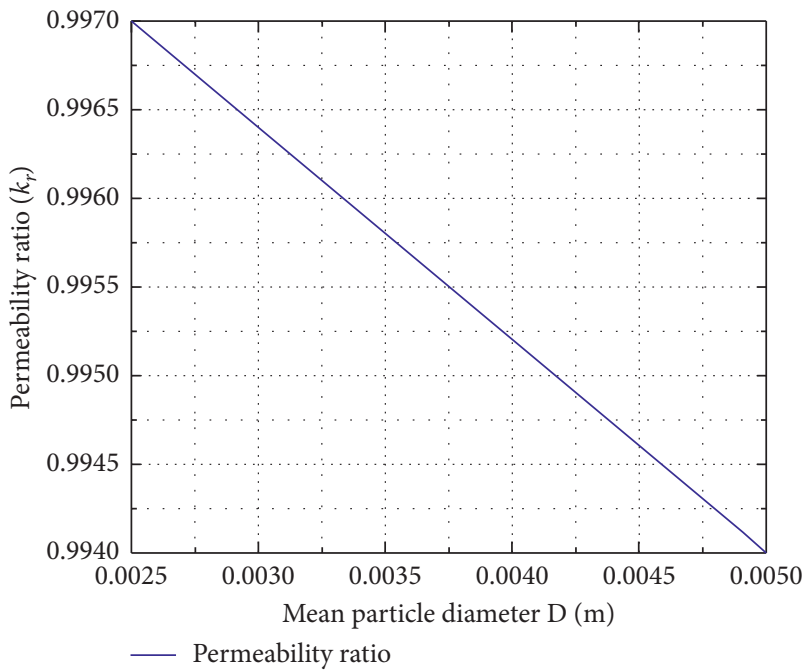

(b)

FIGURE 11: Packed-pipe permeability, sand-pack permeability, and permeability ratio as a function of mean particle diameter: (a) packedpipe permeability and sand-pack permeability as a function of mean particle diameter; (b) permeability ratio as a function of mean particle diameter.

Equation (34) characterizes the relationship between the packed-pipe permeability and the sand-pack properties. The benchmark values of the parameters that are used for conducting sensitivity analysis are as follows: $\gamma=0.8$, $D=0.05 \mathrm{~m}, R=0.01 \mathrm{~m}$, and $\phi=0.2$.

Figure 10(a) shows the packed-pack permeability and sand-pack permeability with different values of $\gamma$. The value of $\gamma$ normally ranges from 0.7 to 1 , and a larger value of $\gamma$ indicates that the particle size is more uniform. In
Figure 10(a), one can find that both the packed-pack permeability and sand-pack permeability are decreased as the value of $\gamma$ is increased. This manifests that a more uniform size of the sand particle can lead to a less packed-pipe and sand-pack permeability. Figure 10(b) shows the permeability ratio $k_{r}$ (see equation (24)) that is calculated with the results in Figure 10(a). A larger value of $k_{r}$ indicates a smaller relative difference between the packed-pipe permeability and the sand-pack permeability. As shown in Figure 10(b), 


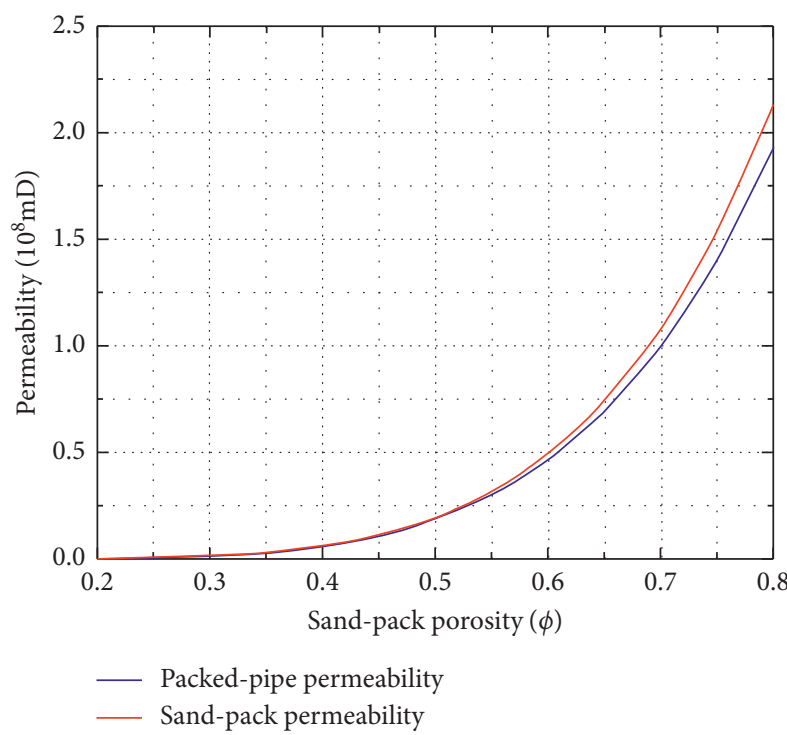

(a)

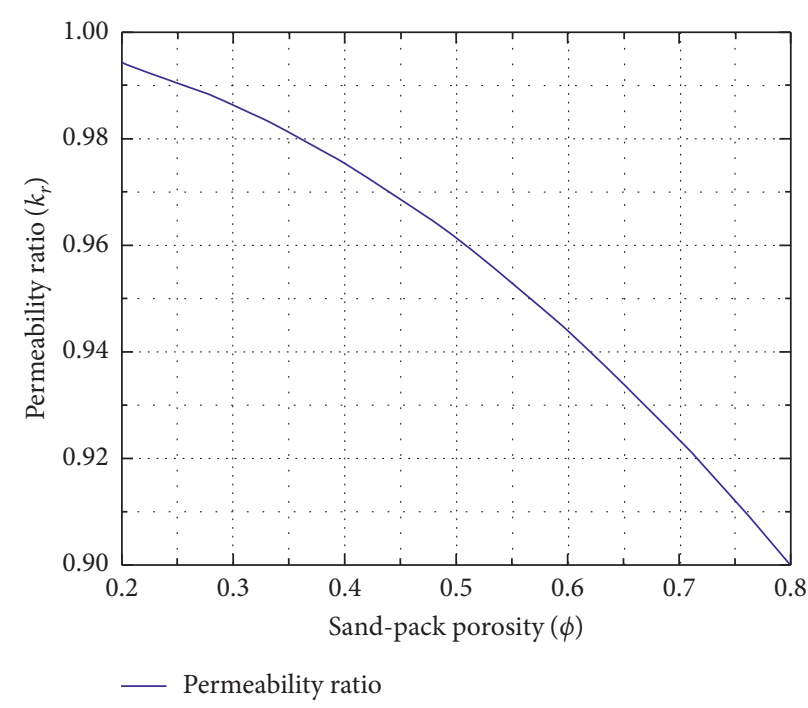

(b)

FIGURE 12: Packed-pipe permeability, sand-pack permeability, and permeability ratio as a function of sand-pack porosity: (a) packed-pipe permeability and sand-pack permeability as a function of sand-pack porosity; (b) permeability ratio as a function of sand-pack porosity.

the permeability ratio is increased as the value of $\gamma$ is increased. However, it is worth noting that as $\gamma$ is varied from 0.7 to $1, k_{r}$ is only slightly increased from 0.9936 to 0.9948 . This implies that the sorting of the sand particles will not induce a large difference between the pack-pipe permeability and the sand-pack permeability.

Figure 11 presents the packed-pipe permeability, sandpack permeability, and permeability ratio as a function of mean particle diameter. In Figure 11(a), it can be observed that the packed-pipe permeability and sand-pack permeability are increased as the mean particle diameter is increased, whereas, in Figure 11(b), the permeability ratio is decreased as the mean particle diameter is increased. In Figure 11(a), the packed-pipe permeability exhibits very similar values to those of the sand-pack permeability. In Figure 11(b), the permeability ratio is slightly less than 1 although it is decreased from 0.9970 to 0.9940 .

Figure 12(a) describes the packed-pipe permeability and sand-pack permeability as a function of sand-pack porosity. As shown in this figure, the packed-pipe permeability and the sand-pack permeability are increased rapidly as the sandpack porosity is increased. Figure 12(b) shows the change of the permeability ratio with different sand-pack porosity. It can be observed that the permeability ratio $k_{r}$ is around 0.9 with $\phi=0.8$, indicating that the difference between and packed-pipe permeability and sand-pack permeability can be significant with large sand-pack porosity.

Comparing the results shown in Figures 10 through 12, one can find that the sand-pack porosity exerts a more significant effect on the packed-pipe permeability and permeability ratio. This is because the sand-pack porosity is highly related to the effective space in the porous media for transmitting the fluid. A higher porosity indicates a larger effective space; hence, the packed pipe permeability expresses a larger permeability with high sand-pack porosity.

\section{Conclusions}

In this work, the authors derived a series of analytical solutions for characterizing the fluid flow through pipes that are packed with sand. These solutions can be used to depict the velocity profiles in a packed-pipe (equation (15)), estimate the flux rate through a packed-pipe (equation (16)), and calculate the equivalent permeability of a packed-pipe (equation (17)). In real applications, one can use these solutions to correct the results of packed-pipe permeability test and characterize the fluid flow in the pore volume of underground reservoirs. For example, in gas hydrate reservoirs, as the gas hydrate is partially decomposed into gas and water in the pore volume, the fluid flow in the space between the undecomposed gas hydrate within the pore volume can be characterized with the proposed solutions. With the aid of these equations, the authors recognized the flow regimes, investigated the velocity profiles, and conducted sensitivity analysis. On the basis of the calculated results, we can form the following conclusions: Poiseuille's law (i.e., equation (4)) is a special form of the derived equivalent permeability solution (i.e., equation (17)); three flow regimes can be observed in a packed-pipe with different values of Darcy parameter, including N-S flow regime, Brinkman flow regime, and Darcy flow regime; a more uniform size of the sand particles can lead to a lower packed-pipe permeability, while a higher permeability ratio and a larger mean particle diameter can lead to a higher packed-pipe permeability and a lower permeability ratio; as the sand-pack porosity is increased, the packed-pipe permeability is increased, while the 
permeability ratio is decreased. In comparison to the sorting and mean particle diameter, the sand-pack porosity can exert a more significant effect on the packed-pipe permeability and permeability ratio.

\section{Nomenclature}

$D_{a}:$ Darcy parameter

$k_{e}$ : Equivalent permeability, $\mathrm{mD}$

$k_{r}$ : Permeability ratio

$k_{s}$ : Permeability of the sand-pack, $\mathrm{mD}$

$L$ : $\quad$ Length of the pipe, $\mathrm{m}$

$p$ : $\quad$ Pressure, $\mathrm{MPa}$

$p_{i n}:$ Average pressure at the inlet surface, $\mathrm{MPa}$

$p_{\text {out }}$ : Average pressure at the outlet surface, $\mathrm{MPa}$

$q$ : $\quad$ Flux rate, $\mathrm{m}^{3} / \mathrm{d}$

$r$ : Cylindrical coordinate, which is along the direction from pipe center to pipe wall

$R: \quad$ Inner radius of the pipe, $m$

$r_{D}$ : Dimensionless radius

$v_{D}$ : Dimensionless velocity

$\beta_{1}$ : Unit conversion factors, 0.0853

$\beta_{2}$ : Unit conversion factors, $1.01 \times 10^{15}$

$\mu$ : Viscosity, $\mathrm{mPa} \cdot \mathrm{s}$

$\nu: \quad$ Velocity, $\mathrm{m} / \mathrm{d}$

$\phi$ : Porosity

\section{Data Availability}

No data were used to support this study.

\section{Conflicts of Interest}

The authors declare that they have no conflicts of interest.

\section{References}

[1] Q.-H. Nguyen, P.-T. Nguyen, and B. Q. Tang, "Energy equalities for compressible Navier-Stokes equations," Nonlinearity, vol. 32, no. 11, pp. 4206-4231, 2019.

[2] Y.-H. Yu and S. A. Kinnas, "Roll response of various hull sectional shapes using a Navier-Stokes solver," International Society of Offshore and Polar Engineers, vol. 19, no. 1, pp. 46-51, 2009.

[3] E. Hagenbach, "Ueber die Bestimmung der Zähigkeit einer Flüssigkeit durch den Ausfluss aus Röhren," Annalen der Physik und Chemie, vol. 185, no. 3, pp. 385-426, 1860.

[4] H. Darcy, Les Fontaines Publiques De La Ville De Dijon, Dalmont, Paris, France, 1856.

[5] G. Q. Chen, L. Zeng, and D. Taylor, "Taylor dispersion in a packed tube," Communications in Nonlinear Science and Numerical Simulation, vol. 14, no. 5, pp. 2215-2221, 2009.

[6] K. Yuki, M. Okumura, H. Hashizume, S. Toda, N. B. Morley, and A. Sagara, "Flow visualization and heat transfer characteristics for sphere-packed pipes," Journal of Thermophysics and Heat Transfer, vol. 22, no. 4, pp. 632-648, 2008.

[7] A. M. Siddiqui, S. Sadiq, and T. Haroon, "Macroscopic analysis of flow through a porous medium between two circular cylinders," Journal of Porous Media, vol. 14, no. 9, pp. 751-760, 2011.
[8] E. T. Hashim and R. S. Karmel, "Pressure drop in a horizontal packed pipe," Petroleum Science and Technology, vol. 31, no. 1-4, pp. 1091-6466, 2013.

[9] Z. Wu, X. Fu, and G. Wang, "Complete spatial concentration distribution for taylor dispersion in packed tube flow," International Journal of Heat and Mass Transfer, vol. 92, pp. 987-994, 2016.

[10] M. Nazififard and K. Y. Suh, "CFD analysis of a sphere-packed pipe for potential application in the molten salt blanket system," Atw-International Journal for Nuclear Power, vol. 61, no. 8-9, pp. 543-547, 2016.

[11] J. Yang, J. Wu, L. Zhou, and Q. Wang, "Computational study of fluid flow and heat transfer in composite packed beds of spheres with low tube to particle diameter ratio," Nuclear Engineering and Design, vol. 300, pp. 85-96, 2016.

[12] H. C. Brinkman, "A calculation of the viscous force exerted by a following fluid on a dense swarm of particles," Applied Scientific Research, vol. A1, pp. 27-34, 1949.

[13] L. Durlofsky and J. F. Brady, "Analysis of the brinkman equation as a model for flow in porous media," Physics of Fluids, vol. 30, no. 11, pp. 3329-3341, 1987.

[14] E. J. Hinch, "An averaged-equation Approach to particle interactions in a fluid suspension," Journal of Fluid Mechanics, vol. 83, no. 4, pp. 695-720, 1977.

[15] I. D. Howells, "Drag due to the motion of a Newtonian fluid through a sparse random array of small fixed rigid objects," Journal of Fluid Mechanics, vol. 64, no. 3, pp. 449-476, 1974.

[16] M. Muthukumar and K. F. Freed, "On the Stokes problem for a suspension of spheres at nonzero concentrations. II. Calculations for effective medium theory," The Journal of Chemical Physics, vol. 70, no. 12, pp. 5875-5887, 1979.

[17] H. A. Belhaj, K. R. Agha, S. D. Butt, and M. R. Islam, "A comprehensive numerical simulation model for non-Darcy flow including viscous, inertial and convective contributions," in Proceedings of Nigeria Annual International Conference and Exhibition, pp. 4-6, Society of Petroleum Engineers, Abuja, Nigeria, August 2003.

[18] Comsol, COMSOL, Comsol, Inc, Burlington, MA, USA, 2019.

[19] R. R. Berg, "Method for determining permeability from reservoir rock properties," Transactions of Gulf Coast Association of Geological Societies, vol. 20, pp. 303-335, 1970. 